\title{
Heterogeneity in reporting on urinary outcome and cure after surgical interventions for stress urinary incontinence in adult neuro-urological patients: A systematic review
}

\author{
Sarah H.M. Reuvers ${ }^{1}$ (D) ｜ Jan Groen' ｜ Jeroen R. Scheepe ${ }^{1} \quad$ Lisette A. 't Hoen' \\ David Castro-Diaz ${ }^{2}$ | Bárbara Padilla-Fernández ${ }^{2}$ (D) | Giulio Del Popolo ${ }^{3}$ \\ Stefania Musco ${ }^{3}$ | Jürgen Pannek ${ }^{4}$ (D) | Thomas M. Kessler ${ }^{5}$ (D) | \\ Marc P. Schneider ${ }^{5}$ | Gilles Karsenty ${ }^{6}$ (D) Veronique Phé $^{7} \mid$ Rizwan Hamid $^{8}$ | \\ Hazel Ecclestone ${ }^{8}$ | Bertil F.M. Blok ${ }^{1}$
}

\footnotetext{
${ }^{1}$ Department of Urology, Erasmus MC, Rotterdam, The Netherlands

${ }^{2}$ Department of Urology, Hospital

Universitario de Canarias, Universidad de

La Laguna, Tenerife, Spain

${ }^{3}$ Department of Neuro-Urology, Careggi

University Hospital, Florence, Italy

${ }^{4}$ Department of Neuro-Urology, Swiss

Paraplegic Center, Nottwil, Switzerland

${ }^{5}$ Department of Neuro-Urology, Spinal

Cord Injury Center \& Research, University

of Zürich, Balgrist University Hospital,

Zürich, Switzerland

${ }^{6}$ Department of Urology, La Conception

Hospital, Assistance Publique-Hôpitaux de

Marseille, Aix-Marseille University,

Marseille, France

${ }^{7}$ Department of Urology, Pitié-Salpêtrière Academic Hospital, Assistance Publique-

Hôpitaux de Paris, Paris 6 University, Paris,

France

${ }^{8}$ Department of Neuro-Urology, London Spinal Injuries Centre, Stanmore, United Kingdom

Correspondence

Sarah H.M. Reuvers, Department of

Urology, Erasmus MC, Rotterdam, The

Netherlands. Wijtemaweg 80, Room Na
}

Aims: To describe all outcome parameters and definitions of cure used to report on outcome of surgical interventions for stress urinary incontinence (SUI) in neurourological (NU) patients.

Methods: This systematic review was performed and reported according to the Preferred Reporting Items for Systematic Reviews and Meta-Analyses (PRISMA) statement. The study protocol was registered and published (CRD42016033303; http://www.crd.york.ac.uk/PROSPERO). Medline, Embase, Cochrane controlled trials databases, and clinicaltrial.gov were systematically searched for relevant publications until February 2017.

Results: A total of 3168 abstracts were screened. Seventeen studies reporting on SUI surgeries in NU patients were included. Sixteen different outcome parameters and nine definitions of cure were used. Six studies reported on objective outcome parameters mainly derived from urodynamic investigations. All studies reported on one or more subjective outcome parameters. Patient-reported pad use (reported during interview) was the most commonly used outcome parameter. Only three of 17 studies used standardized questionnaires (two on impact of incontinence and one on quality of life). Overall, a high risk of bias was found.

Conclusions: We found a considerable heterogeneity in outcome parameters and definitions of cure used to report on outcome of surgical interventions for SUI in NU patients. The results of this systematic review may begin the dialogue to a future consensus on this topic. Standardization of outcome parameters and definitions of

Hashim Hashim led the peer-review process as the Associate Editor responsible for the paper.

This is an open access article under the terms of the Creative Commons Attribution-NonCommercial License, which permits use, distribution and reproduction in any medium, provided the original work is properly cited and is not used for commercial purposes.

(C) 2017 The Authors. Neurourology and Urodynamics Published by Wiley Inc. 
1724, 3015 CN Rotterdam, The

Netherlands.

Email: s.reuvers@erasmusmc.nl; sarahreuvers@yahoo.com

Funding information

European Association of Urology cure would enable researchers and clinicians to consistently compare outcomes of different studies and therapies.

\section{K E Y W O R D S}

neurogenic, outcome assessment, patient reported outcome measures, stress urinary incontinence,

treatment outcome, urinary bladder, urinary incontinence

\section{1 | INTRODUCTION}

Patients with neurological disease may show various urological symptoms, depending on the type of disease and the neurological location of the lesion. ${ }^{1,2}$ Both storage and voiding problems can considerably reduce patients quality of life. ${ }^{3}$ An impaired neurological control of the external sphincter may be the cause of stress urinary incontinence (SUI), defined as urinary incontinence that occurs on exertion, effort, sneezing, or coughing. ${ }^{4}$ This bothersome condition affects many neuro-urological (NU) patients, typically those with a meningomyelocele or a conus-cauda equina lesion. ${ }^{1}$ Owing to the fact that SUI in NU patients often occurs together with other urological dysfunction such as detrusor overactivity and reduced bladder compliance, ${ }^{1,3}$ treatment of SUI in NU patients requires a specific approach. Moreover, NU patients may perceive bother from urinary incontinence differently compared to non-NU patients due to altered sensation and impaired mobility. Therefore, the outcome parameters and the definitions of success or cure used to report on outcome of surgical interventions for SUI in NU patients require specific attention.

To identify the most appropriate therapy, studies on the outcomes of the different therapies used to treat SUI in NU patients should ideally be reported in a standardized way. We performed a systematic review to describe all urinary parameters and definitions of success or cure used to report on outcome of surgical interventions for SUI in NU patients.

\section{2 | MATERIALS AND METHODS}

\subsection{Study registration}

The study protocol was registered and published on PROSPERO (CRD42016033303) (http://www.crd.york.ac. uk/PROSPERO). This systematic review was performed and reported according to the Preferred Reporting Items for Systematic Reviews and Meta-Analyses (PRISMA) statement $^{5}$ and Cochrane Handbook for Systematic Reviews of Interventions. ${ }^{6}$

\section{2 | Literature search}

The Medline, Embase, Cochrane controlled trials databases, and clinicaltrial.gov were systematically searched for all relevant publications until February 2017. The search strategy is available in Supplementary Material S1. Duplicates were removed. No date restrictions were applied. Non-English texts were excluded. Additionally, reference lists of relevant reviews were hand-searched for missed relevant articles.

\section{3 | Study selection}

Our aim was to include all publications of original studies that used a predefined urinary outcome parameter or a definition of success or cure to report on outcome of surgical interventions for SUI in adult NU patients. Conference abstracts, reviews, and case series with $<10$ NU patients were excluded. Reviews served only to check the references for eligible extra articles. Studies with both adult NU and non-NU patients or with both children and adult NU patients were included only if adult NU patients were separately reported on or if $>90 \%$ of the study population were adult NU patients.

Endnote (EndNote X7, Thomson Reuters, 1500 Spring Garden Street, Fourth Floor, Philadelphia, PA 19130) was used to store identified abstracts and to sort the abstracts for inclusion and exclusion. Each title and abstract was reviewed for eligibility by two out of four reviewing authors (BB, JG, JS, SR) independently. Articles of which the abstract met the eligibility criteria were reviewed in full text. Full text selection was performed by two authors independently (JG, SR) using a standardized screening form. Discrepancy between the two authors was resolved by discussion or by consulting a third reviewer (BB). We reported on the literature search and study selection in a PRISMA flow diagram. ${ }^{5}$

\section{$2.4 \mid$ Outcomes}

All urinary outcome parameters and definitions of cure or success used to report on outcome of surgical interventions 
for SUI in adult NU patients were summarized. Outcome parameters containing information from questionnaires and patient interviews were considered subjective outcome parameters. Outcome parameters were considered objective when derived from bladder diaries, pad tests, cough stresstests, or urodynamic investigations.

\subsection{Data extraction and risk of bias assessment}

Data on general study characteristics were retrieved by the first author and checked by JG. Two authors (SR and JG) independently extracted predefined data from the included publications using a standardized data extraction form. A risk of bias analysis for included non-randomized comparative studies was performed by using the Cochrane Risk of bias Assessment $\mathrm{Tool}^{7}$ in combination with an assessment of the main confounders following the recommendations of the Cochrane handbook for non-randomized comparative studies. ${ }^{6} \mathrm{~A}$ list of the main confounders was developed and a priori agreed on with clinical content experts (EAU Neuro-Urology guidelines panel). Identified confounders were age, gender, mixed versus stress incontinence, underlying NU pathology, perineal sensation, previous treatments for SUI, and previous pelvic surgeries. Confounders were determined for the studies during data extraction. The confounding bias was classified as "high" if the confounder was not considered or described, was imbalanced between the groups or was unadjusted during analysis. The risk of bias in non-comparative studies was determined by assessing the attrition bias (incomplete outcome data), the reporting bias (selective outcome reporting), and availability of an a priori protocol. External validity of these studies was reported by assessing whether participants were selected consecutively. This is a pragmatic approach based on methodological literature. ${ }^{8,9} \mathrm{In}$ addition, the main confounders were assessed for these studies. The risk of bias figure was computed in Review Manager (RevMan) version 5.3 (Copenhagen: The Nordic Cochrane Centre, The Cochrane Collaboration, 2014).

\section{6 | Subgroup analyses}

Subgroup analyses were intended to be performed if there would be sufficient data. Predefined subgroups were men versus women, SUI versus mixed UI, underlying NU pathology, and no versus one/more former surgeries with potential effect on continence.

\section{3 | RESULTS}

\section{1 | Search results}

Figure 1 shows the PRISMA flow diagram of the literature search and study selection. After screening of 3168 abstracts,
182 full texts were reviewed. Finally, 17 studies were included in this systematic review. ${ }^{10-26}$

\subsection{Characteristics of included studies}

The included studies were published between 1995 and 2017 and report the results of various SUI surgeries. Table 1 shows the descriptives of the included studies. Most studies had a retrospective single-arm study design. With one exception, all studies were single-center studies. Twelve studies reported on NU patients only. A total of $452 \mathrm{NU}$ patients were included in the studies. Most studies included mixed patient populations regarding underlying NU pathology, detrusor overactivity, mixed urinary incontinence and pure SUI, and patients with and without previous SUI, and other pelvic surgeries.

\section{3 | Results on outcome parameters}

Table 2 shows the outcome parameters used per study. In total, 16 different outcome parameters were used in the 17 included studies. Furthermore categorization of the outcomes differed (eg, patient-reported leakage/continence). Eleven studies had applied two or more outcome parameters. Six of the 17 studies reported on both an objective and a subjective outcome parameter.

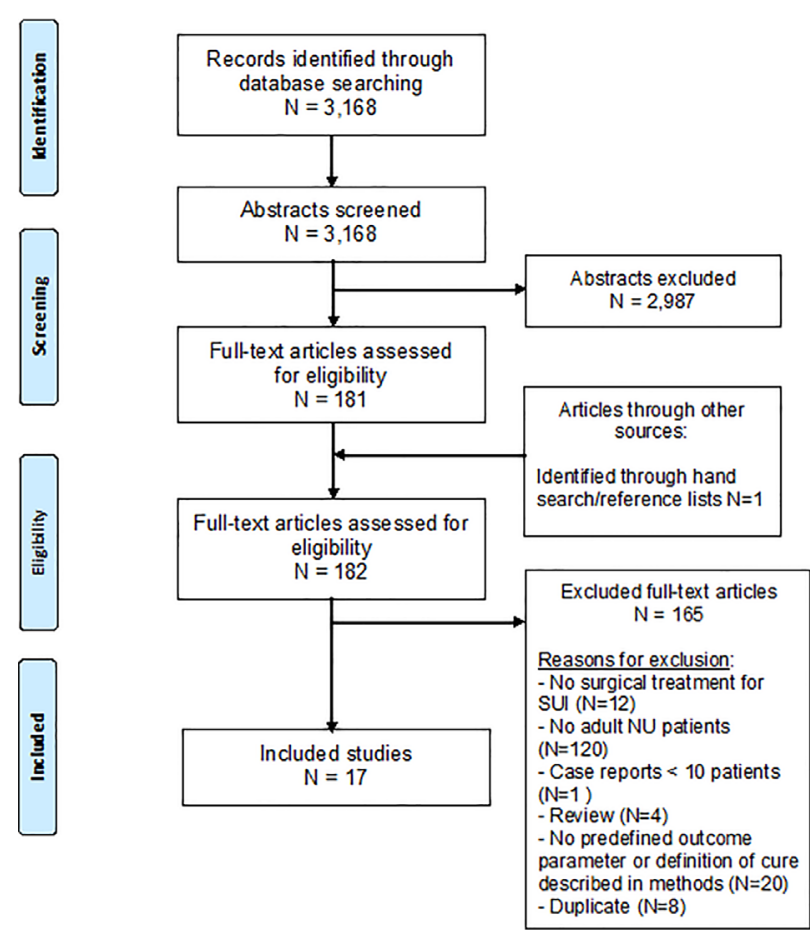

FIGURE 1 PRISMA flow diagram of identified, excluded, and included studies. NU, neuro-urological; SUI, stress urinary incontinence 

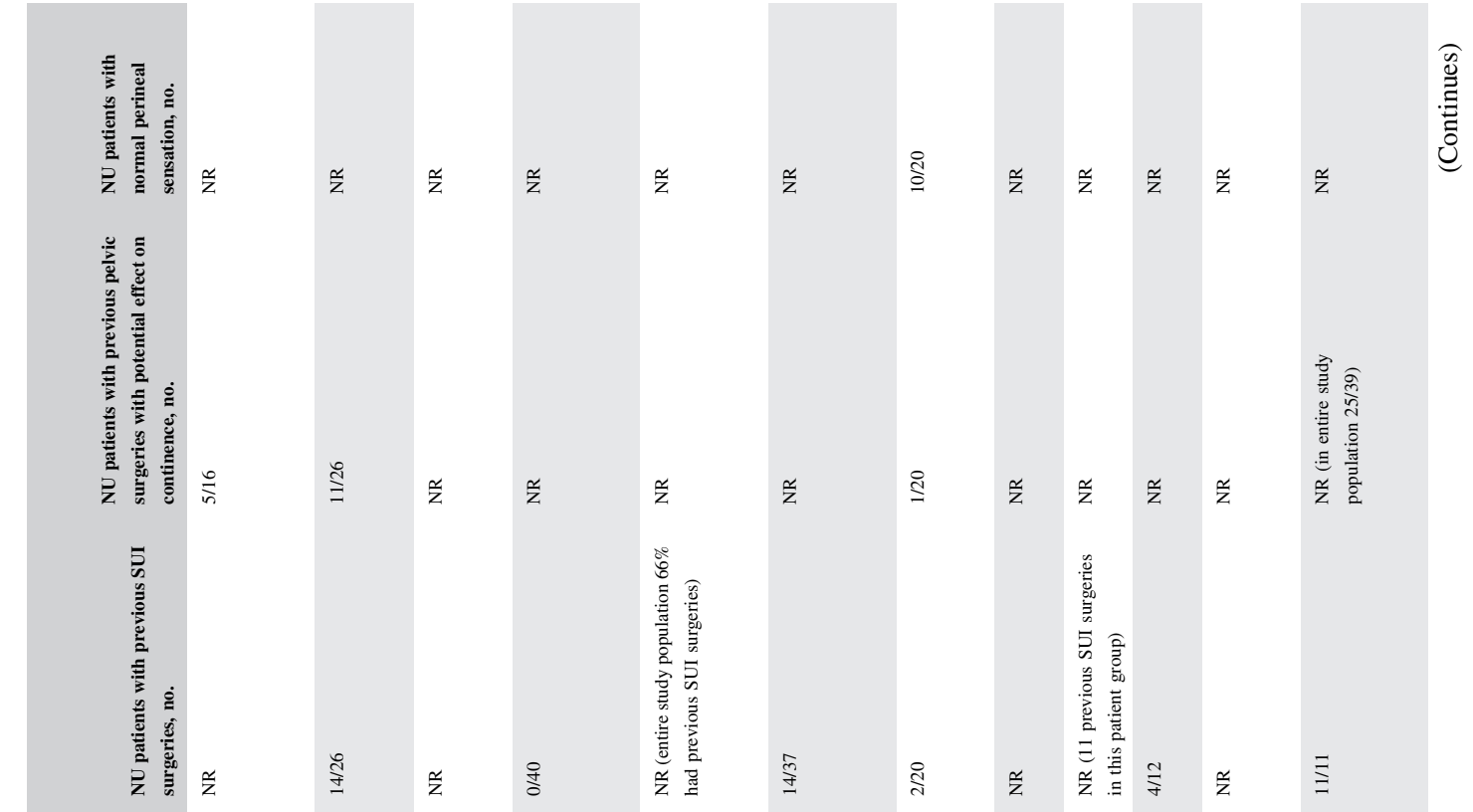

II

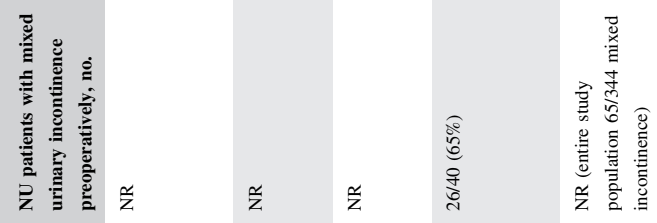

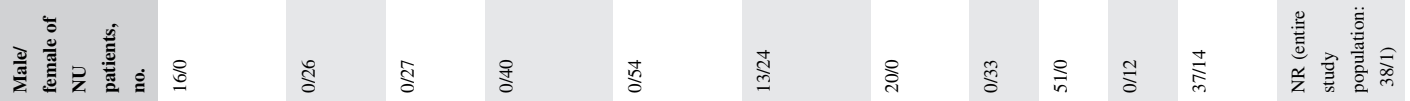

M 1 llm

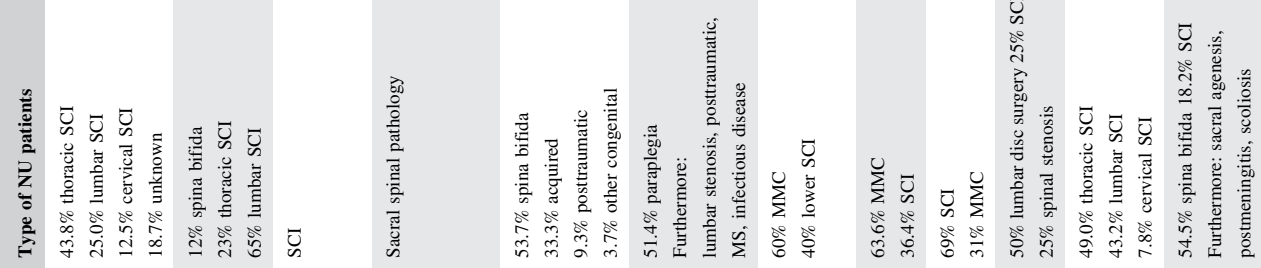

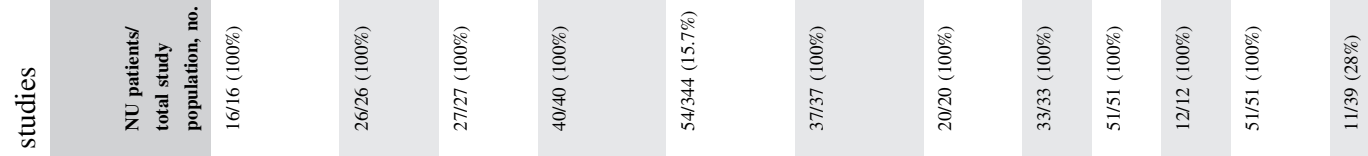

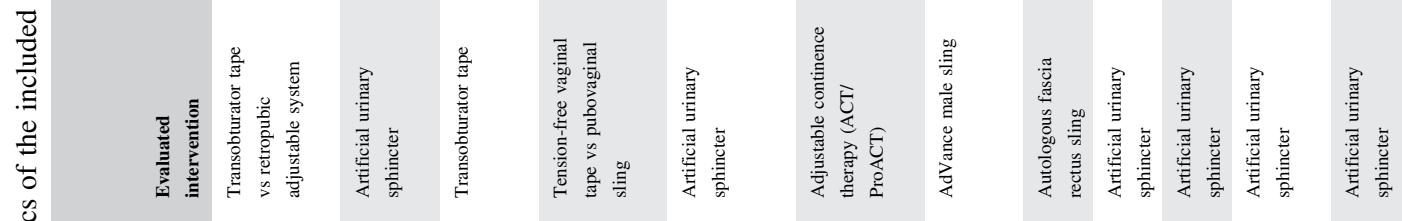

HIH HU II MHHW

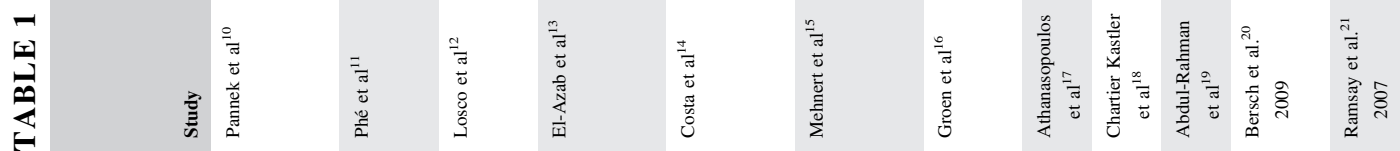




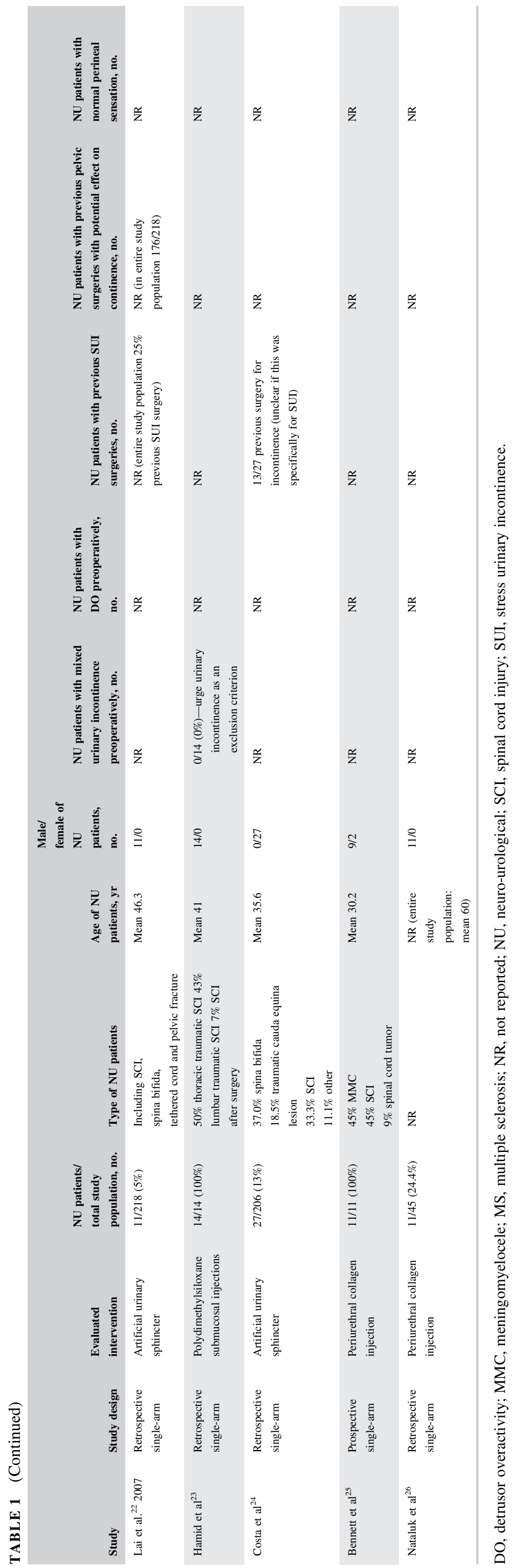




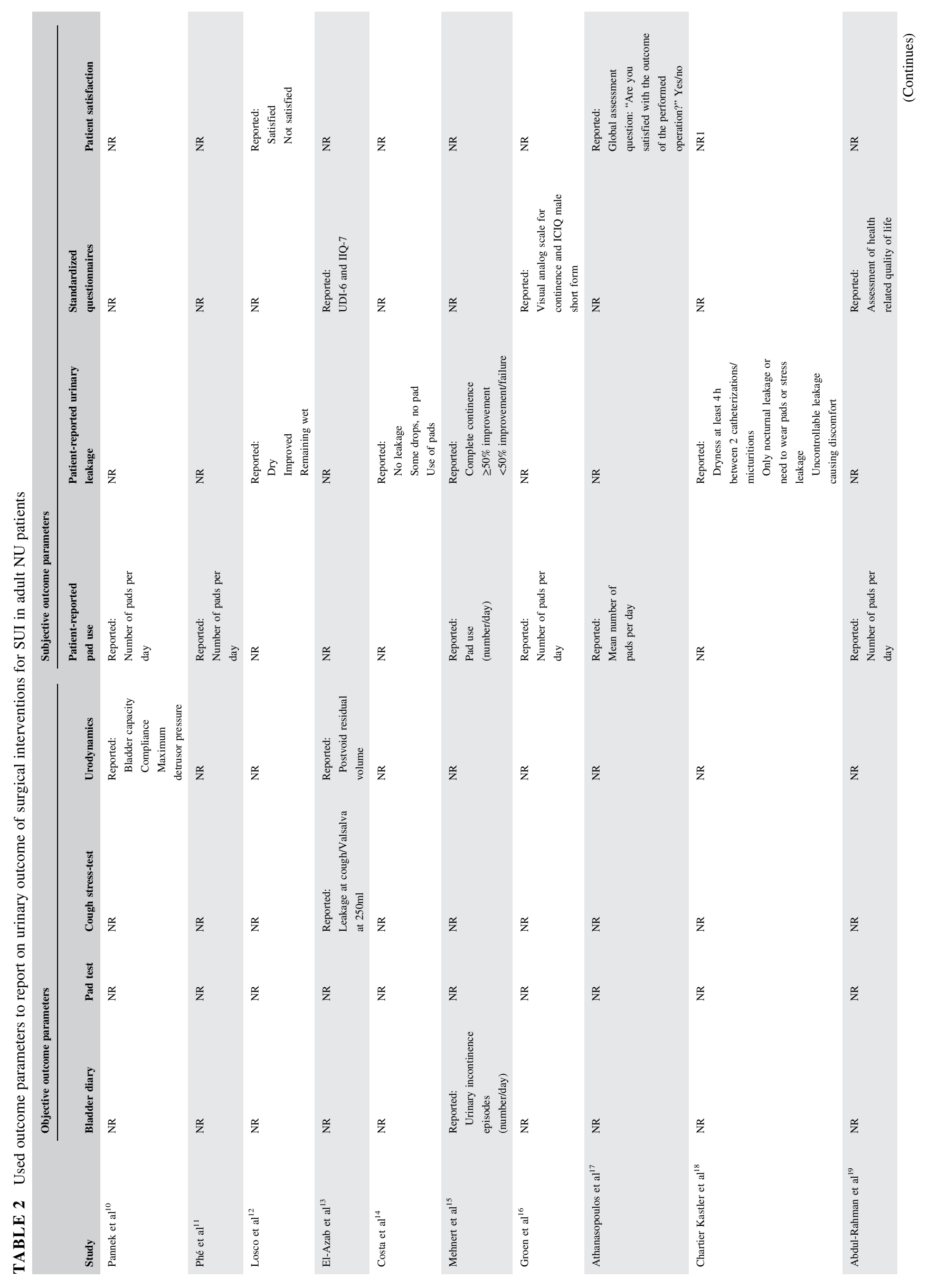


560

REUVERS ET AL.

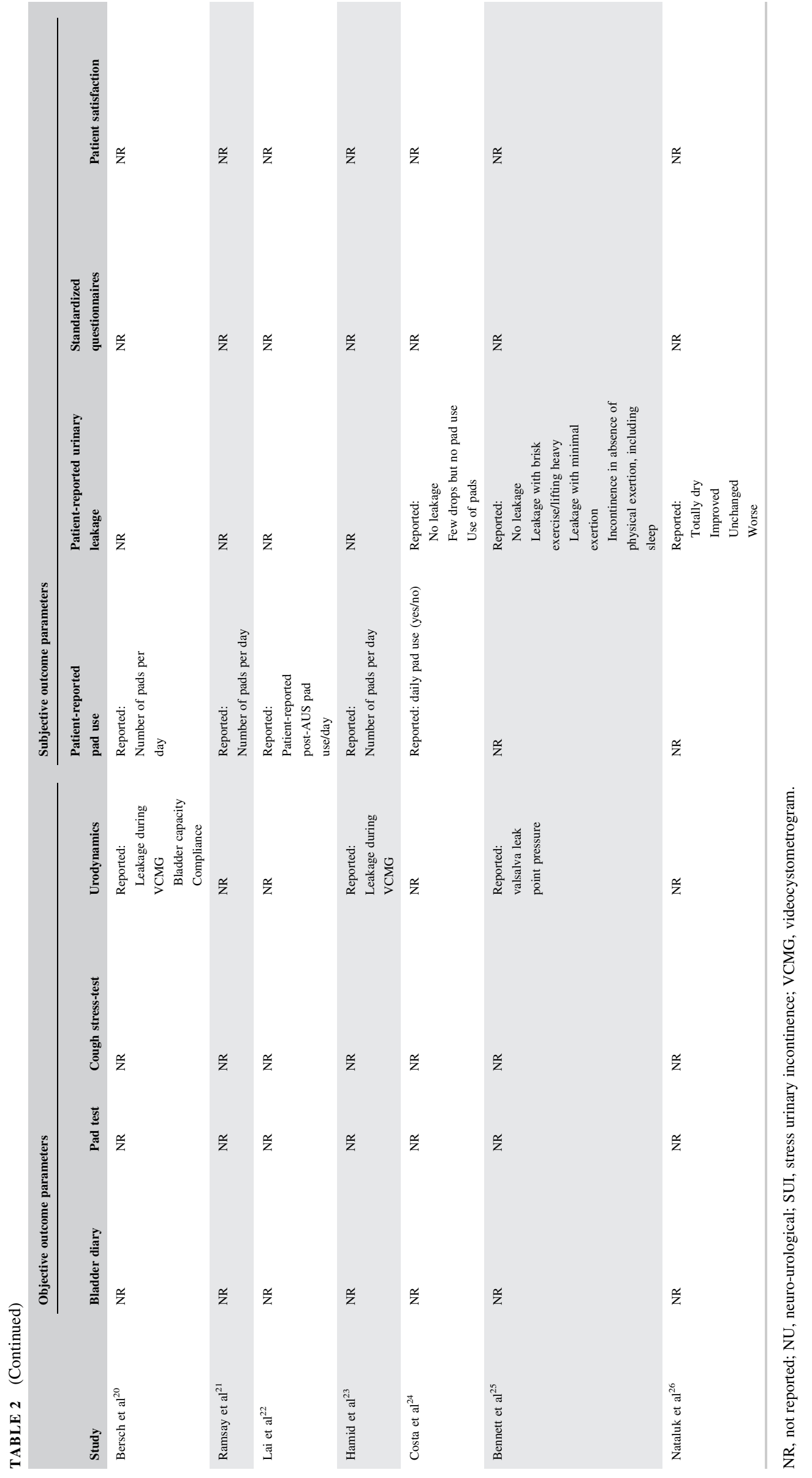


TABLE 3 Used definitions of cure or continence to report on success of surgical interventions for SUI in adult NU patients

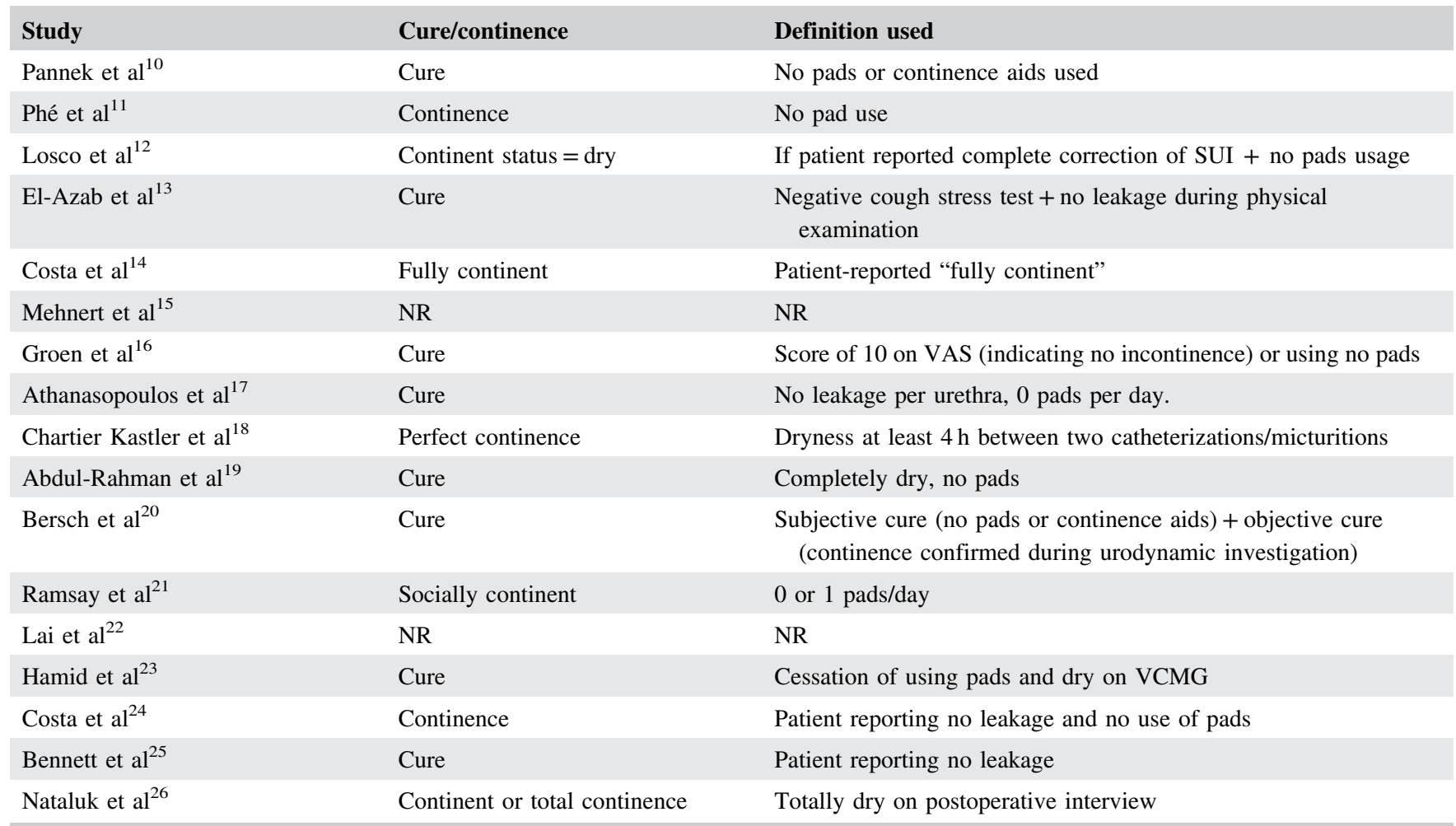

NR, not reported; NU, neuro-urological; SUI, stress urinary incontinence; VCMG, videocystometrogram.

\subsubsection{Objective outcome parameters}

Six of 17 (35.3\%) included studies reported on objective outcome parameters. Pad tests were not reported on. In one study, patients used a bladder diary to report the number of urinary incontinence episodes per day. One study reported the results of a cough stress-test. Urodynamics was the most used investigation to measure an objective outcome parameter; that is, in five studies. Bladder capacity, compliance, maximum detrusor pressure, postvoid residual volume, leakage during videocystometrogram, and Valsalva leak point pressure were the objective outcome parameters that were derived from urodynamic investigations.

\subsection{2 | Subjective outcome parameters}

Patient-reported pad use (number of pads/24h or yes/no daily pad use reported during an interview) was the most utilized outcome parameter; used in eleven studies. Three studies applied standardized questionnaires. In seven studies patients reported on their urinary leakage status in a post-intervention interview. Two studies reported on patient satisfaction.

\subsection{Results on definition of success or cure}

Table 3 provides an overview of the different definitions for cure or continence used. Fifteen of 17 studies reported on such a definition. In these 15 studies, nine different definitions were used. Only two of five studies that reported on cure and used an objective and a subjective outcome parameter, used a combination of both outcomes to define cure.

\section{5 | Subgroup analyses}

It was not contributive or possible to perform subgroup analyses. First, the number of included studies was small; second, because most studies identified included mixed populations (gender, underlying NU pathology, SUI, and mixed UI, former surgeries with potential effect on continence); and finally, subanalyses and information on predefined groups was often missing (Table 1).

\section{6 | Risk of bias assessment}

Most of the included studies were assessed as having high or unclear risk of bias (Figure 2). In most retrospective studies, it was unclear if an a priori protocol was available and if there was selective outcome reporting. In one third of these studies, it was unclear if there were incomplete outcome data. Most studies included study participants consecutively. The two comparative studies had a high risk of bias for most assessed factors of the Cochrane Risk of bias Assessment Tool and the confounding factors. 


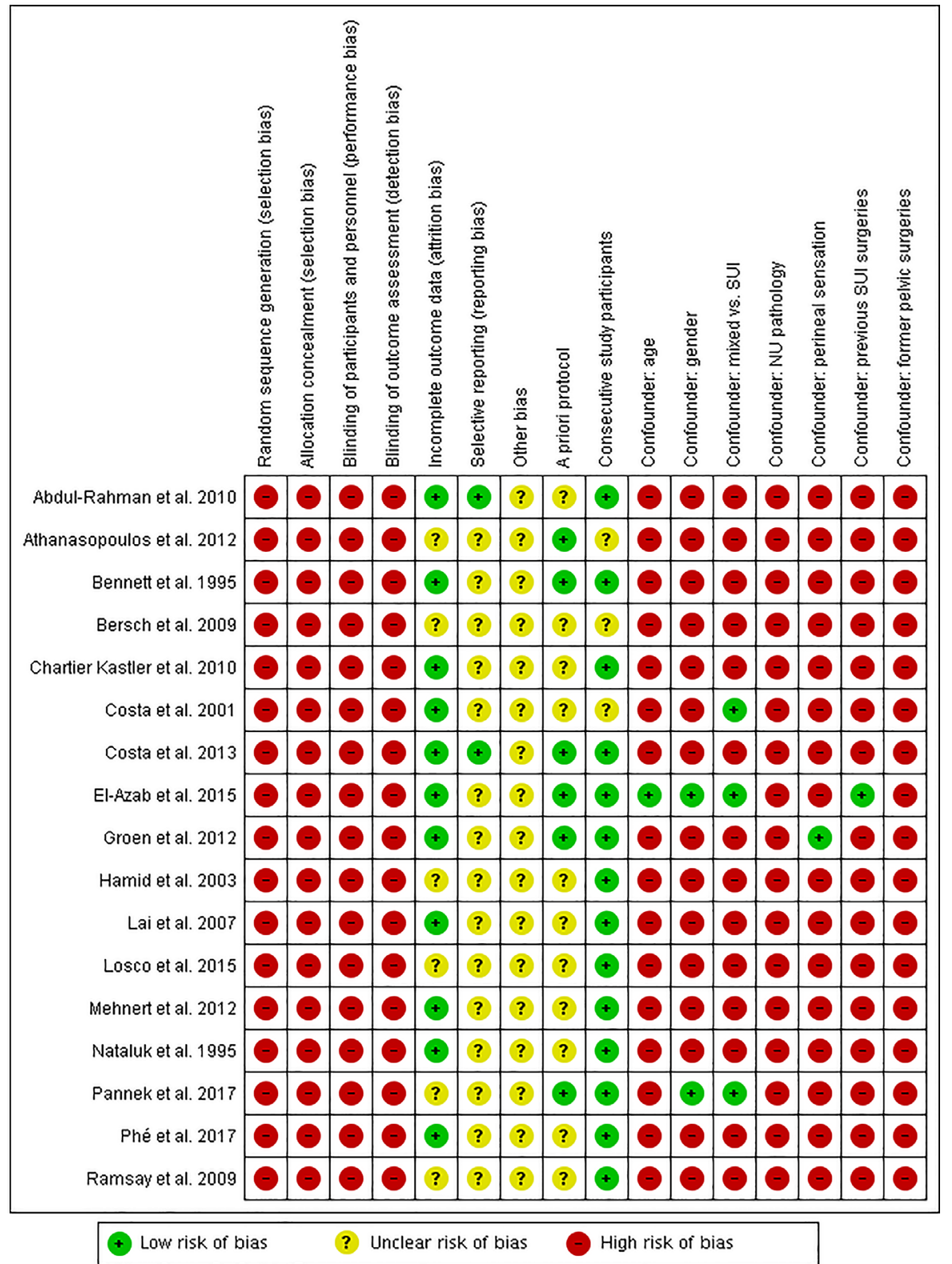

FIGURE 2 Risk of bias summary. NU, neuro-urological; SUI, stress urinary incontinence

\section{4 | DISCUSSION}

\section{1 | Principal findings}

In this systematic review, we have presented all parameters and definitions of cure to report on the outcome of surgical interventions for SUI in adult NU patients. Sixteen different outcome parameters and nine different definitions of cure or continence were used. Most outcomes and definitions of cure were based on non-standardized patient self-assessments (of pad use per day or leakage/continence). A minority of studies made use of objective outcome parameters or validated questionnaires. To the best of our knowledge, this is the first systematic review on this topic in this specific patient group. It is evident that there is a considerable heterogeneity in the urinary outcome parameters and definitions of cure used to report on outcome of surgical interventions for SUI in adult NU patients.

\section{2 | Findings in the context of the existing evidence}

The heterogeneity of outcome reporting makes it more difficult to interpret and compare different studies and therapies. The Core Outcome Measures in Effectiveness Trials (COMET) initiative supports the development of standardized sets of outcomes in all fields of health research. ${ }^{27}$ In the field of urology such core outcome sets are available for prostate cancer and male sexual dysfunction, but not for UI. The 
International Continence Society (ICS) and the International Consultation on Incontinence (ICI) recommend using both objective and subjective outcome parameters in UI research. ${ }^{28,29}$ Despite this, these organizations do not provide a definition of cure or make recommendations for the specific outcome parameters to be used.

Specifically, in the field of NU patients undergoing SUI surgery there is no consensus on outcome parameters. The ICS does not provide a recommendation on this topic for research in NU patients. The ICI recommends using changes in detrusor leak point pressure for research purposes in NU patients if appropriate. ${ }^{28}$ Nevertheless, this parameter was not used in any of the included studies in our systematic review. The EAU guidelines mention prevention of deterioration of the upper urinary tract and optimization of the quality of life as the most important urological treatment goals for NU patients. ${ }^{30}$ Therefore, we would expect urodynamic investigations and quality of life measures to be used more often in this patient group. NU patients may have altered sensation and impaired mobility and consequently perceive (UI) complaints different than to non-NU patients. Thus, measuring patients' perception of UI complaints and their healthrelated quality of life (rather than quantifying symptoms) is important, especially in this patient group. Phé et $\mathrm{al}^{31}$ and Castillo et $\mathrm{al}^{32}$ reported in their reviews on the commonly used outcome parameters and definitions of cure or treatment success used after SUI surgery (not specifically on NU patients). Phé et $\mathrm{al}^{31}$ reviewed publications on all SUI surgeries from 1995 to 2014 and Castillo et $\mathrm{al}^{32}$ focused on publications on female SUI from 2005 and 2006. In our systematic review, we found that five out of 17 (29.4\%) included studies used outcome parameters derived from urodynamic investigations. Phé et $\mathrm{al}^{31}$ and Castillo et $\mathrm{al}^{32}$ found that urodynamic investigations were performed in 12 of 54 studies (22.2\%) and in 37 of 92 studies (40.2\%), respectively. Only two of the $17(11.8 \%)$ studies in our review applied questionnaires on the impact of UI and only one study used quality-of-life assessments. The questionnaires administered were the UDI-6, IIQ-7, visual analog scale for continence, and ICIQ male short form. These are validated questionnaires, but not specifically for NU patients. Although validated (disease-specific) quality of life questionnaires such as the (SF-)Qualiveen ${ }^{33,34}$ have been introduced in the recent past, they have not always been available. In the review by Phé et $\mathrm{al}^{31}$ validated questionnaires (including quality of life measures) were used in $55.6 \%$ and in the review by Castillo et $\mathrm{al}^{32}$ validated questionnaires were used in $40.2 \%$ and quality of life measures were used in $60.9 \%$ of the studies. So contrary to our expectations, urodynamic investigations and quality of life measures were not used more often in our systematic review in NU patients. For retrospective studies, only available measures from clinical practice can be used. The high number of retrospective studies in our systematic review compared to Phé et $\mathrm{al}^{31}$ and Castillo et $\mathrm{al}^{32}$ could explain the different findings. On the other hand, one would expect quality of life measures and urodynamic investigations as standard of care in NU patients.

Despite the ICS and ICI recommendations, in only six of 17 (35.3\%) included studies in this systematic review both a subjective and an objective outcome parameter was used and only two of these studies used a combination of these parameters to define cure. Compared to the reviews of Phé et $\mathrm{al}^{31}$ and Castillo et $\mathrm{al}^{32}$ in non-neurological patients, where about half of the studies reported on both a subjective and an objective outcome, this number is low. The high number of retrospective studies could again be an explanation for this finding. Comparable to our results, in the reviews of Phé et $\mathrm{al}^{31}$ and Castillo et al ${ }^{32}$ a minority of studies used a combination of subjective and objective outcome parameters to define cure.

Pad use reported by the patient during an interview was the most used outcome parameter in the studies included in our systematic review. Phé et $\mathrm{al}^{31}$ reported on this outcome for some studies, but not structurally for all and Castillo et al.$^{32}$ did not mention this outcome parameter in their review. In one included study ${ }^{17}$ of our review this outcome parameter was chosen because it would reflect the quality of life, referring to a publication by Stoffel et $\mathrm{al}^{35}$ that found a correlation between patient-reported pad use and the impact of UI on quality of life. In other publications the reason for choosing this outcome parameter is not clear, but might be the ease of collecting this information (especially for retrospective series) for both patient and researcher; in addition it does not interfere in a patient's "normal daily voiding routine" (as a bladder diary might do). It is questionable if patient-reported pad use during an interview reflects the quantity of urine $\operatorname{lost}^{36}$ specifically for NU patients with altered sensation in whom the use of incontinence pads is often discouraged to prevent skin problems. Furthermore, it is unknown if patient-reported pad use is comparable to bladder diary reported pad use. As using this outcome parameter may be advantageous, we suggest to further investigate this outcome parameter on psychometric properties, such as test-retest reliability, correlation with bladder diary reported pad use, quantity of urine lost, and quality of life.

\section{3 | Implication for research and clinical practice}

Farag et $\mathrm{al}^{37}$ reported on the success rates of surgical treatments for SUI in both adult and pediatric NU patients in a systematic review. Farag et $\mathrm{al}^{37}$ compared the combined success rates of the included studies on urethral bulking agents to urethral sling procedures and artificial urinary sphincters. These studies however used variable definitions of success. A consistent comparison of the outcomes of therapy can only be made after standardization of outcome parameters and definitions of cure or success. We therefore recommend developing a core 
outcome set for use in UI research with NU patients. It is important that not only medical experts, but also patients and caregivers will be involved in the development of this outcome set, in order to include the various perspectives and also to increase the willingness to implement the outcome set. Until such a set has been developed, we recommend using an objective and a subjective outcome parameter and the combination of both to define cure. Because of the importance of the quality of life, specifically in NU patients, we recommend the use of a disease-specific quality of life questionnaire or a bother questionnaire validated for $\mathrm{NU}$ patients such as the (SF-)Qualiveen ${ }^{33,34}$ as a subjective outcome parameter. Implementing such questionnaires in both research and clinical practice places a focus on optimization of the quality of life for these patients and makes it possible to compare outcomes of different studies. A clear recommendation for the use of a specific objective parameter is not feasible because there is insufficient scientific evidence on the psychometric properties of the different objective measures (bladder diaries, urodynamics, and pad tests), specifically regarding NU patients. ${ }^{38}$

\section{4 $\mid$ Strengths and limitations}

Performing this systematic review, we followed the recommended Cochrane ${ }^{6}$ and PRISMA guidelines. ${ }^{5}$ Our study gives a clear overview of all used urinary parameters and definitions of success or cure to report on the outcome of surgical interventions for SUI in NU patients, and will hopefully begin the dialogue to a future consensus on this topic. Unfortunately, the included studies were primarily retrospective and of poor scientific quality. Furthermore, subgroup analyses were not possible due to the limited number of included studies.

\section{5 | CONCLUSIONS}

This is the first systematic review that has evaluated the various urinary parameters and definitions of cure to report on outcome after surgery for SUI in adult NU patients. We found a considerable heterogeneity in used outcome parameters and definitions of cure. As it is difficult to interpret and compare the outcomes of different therapies as investigators use different reporting systems of outcomes and definitions of cure, the results of this study will hopefully begin the dialogue to a future consensus on this topic.

\section{ACKNOWLEDGMENTS}

We would like to thank Cathy Yuan, for performing the literature search and Karin Plass for providing the full-text articles. Furthermore, we would like to thank Steven MacLennan, Richard Sylvester, and Thomas Lam, methodological committee of the European Association of Urology
Guidelines Office, for their methodological support. The review was supported by the European Association of Urology. The European Association of Urology had no role in the design and conduct of the study; collection, management, analysis, and interpretation of the data; preparation, review, or approval of the manuscript; and decision to submit the manuscript for publication.

\section{ORCID}

Sarah H.M. Reuvers (D) http://orcid.org/0000-0003-14768865

Bárbara Padilla-Fernández (iD) http://orcid.org/0000-00028566-6033

Jürgen Pannek (D) http://orcid.org/0000-0002-9910-1295

Thomas M. Kessler (D) http://orcid.org/0000-0002-1991-

5919

Gilles Karsenty (iD http://orcid.org/0000-0002-9047-3332

\section{REFERENCES}

1. Madersbacher $\mathrm{H}$. The various types of neurogenic bladder dysfunction: an update of current therapeutic concepts. Paraplegia. 1990;28:217-229.

2. Panicker JN, Fowler CJ, Kessler TM. Lower urinary tract dysfunction in the neurological patient: clinical assessment and management. Lancet Neurol. 2015;14:720-732.

3. Vodusek DB. Lower urinary tract and sexual dysfunction in neurological patients. Eur Neurol. 2014;72:109-115.

4. Abrams P, Cardozo L, Fall M, et al. The standardisation of terminology of lower urinary tract function: report from the standardisation sub-committee of the International Continence Society. Neurourol Urodyn. 2002;21:167-178.

5. Moher D, Liberati A, Tetzlaff J, Altman DG, Group P. Preferred reporting items for systematic reviews and meta-analyses: the PRISMA statement. PLoS Med. 2009;6:e1000097.

6. Higgins JPT, Green S, eds. Cochrane handbook for systematic reviews of interventions Version 5.1.0. [updated March 2011]. The Cochrane Collaboration; 2011. Available at: http://handbook.cochrane.org

7. Higgins JP, Altman DG, Gotzsche PC, et al. The Cochrane Collaboration's tool for assessing risk of bias in randomised trials. BMJ. 2011;343:d5928.

8. Dalziel K, Round A, Stein K, Garside R, Castelnuovo E, Payne L. Do the findings of case series studies vary significantly according to methodological characteristics? Health Technol Assess. 2005;9:1-146.

9. Viswanathan M, Ansari MT, Berkman ND, et al. Assessing the risk of bias of individual studies in systematic reviews of health care interventions. Methods Guide for Comparative Effectiveness Reviews. Rockville, MD: Agency for Healthcare Research and Quality; 2012. https://www.ncbi.nlm.nih.gov/books/NBK91433/.

10. Pannek J, Wollner J. Treatment of stress urinary incontinence in men with spinal cord injury: minimally invasive $=$ minimally effective? Spinal Cord. 2017. https://doi.org/10.1038/sc.2017.16 [Epub ahead of print].

11. Phe V, Leon P, Granger B, et al. Stress urinary incontinence in female neurological patients: long-term functional outcomes after 
artificial urinary sphincter (AMS 800TM) implantation. Neurourol Urodyn. 2017;36:764-769.

12. Losco GS, Burki JR, Omar YA, Shah PJ, Hamid R. Long-term outcome of transobturator tape (TOT) for treatment of stress urinary incontinence in females with neuropathic bladders. Spinal Cord. 2015;53:544-546.

13. El-Azab AS, El-Nashar SA. Midurethral slings versus the standard pubovaginal slings for women with neurogenic stress urinary incontinence. Int Urogynecol J. 2015;26:427-432.

14. Costa P, Poinas G, Naoum K Ben, et al. Long-term results of artificial urinary sphincter for women with type III stress urinary incontinence. Eur Urol. 2013;63:753-758.

15. Mehnert U, Bastien L, Denys P, et al. Treatment of neurogenic stress urinary incontinence using an adjustable continence device: 4-year followup. J Urol. 2012;188:2274-2280.

16. Groen LA, Spinoit AF, Hoebeke P, Van Laecke E, De Troyer B, Everaert $\mathrm{K}$. The AdVance male sling as a minimally invasive treatment for intrinsic sphincter deficiency in patients with neurogenic bladder sphincter dysfunction: a pilot study. Neurourol Urodyn. 2012;31:1284-1287.

17. Athanasopoulos A, Gyftopoulos K, McGuire EJ. Treating stress urinary incontinence in female patients with neuropathic bladder: the value of the autologous fascia rectus sling. Int Urol Nephrol. 2012;44:1363-1367.

18. Chartier Kastler E, Genevois S, Game X, et al. Treatment of neurogenic male urinary incontinence related to intrinsic sphincter insufficiency with an artificial urinary sphincter: a French retrospective multicentre study. Bju Int. 2011;107:426-432.

19. Abdul-Rahman A, Attar KH, Hamid R, Shah PJ. Long-term outcome of tension-free vaginal tape for treating stress incontinence in women with neuropathic bladders. Bju Int. 2010;106:827-830.

20. Bersch U, Gocking K, Pannek J. The artificial urinary sphincter in patients with spinal cord lesion: description of a modified technique and clinical results. Eur Urol. 2009;55:687-693.

21. Ramsay AK, Granitsiotis P, Conn IG. The use of the artificial urinary sphincter in the West of Scotland: a single centre 10-year experience. Scott Med J. 2007;52:14-17.

22. Lai HH, Hsu EI, Teh BS, Butler EB, Boone TB. 13 years of experience with artificial urinary sphincter implantation at Baylor College of Medicine. J Urol. 2007;177:1021-1025.

23. Hamid R, Arya M, Khastgir J, Patel HR, Shah PJ. The treatment of male stress urinary incontinence with polydimethylsiloxane in compliant bladders following spinal cord injury. Spinal Cord. 2003;41:286-289.

24. Costa P, Mottet N, Rabut B, Thuret R, Ben Naoum K, Wagner L. The use of an artificial urinary sphincter in women with type III incontinence and a negative Marshall test. J Urol. 2001;165:1172-1176.

25. Bennett JK, Green BG, Foote JE, Gray M. Collagen injections for intrinsic sphincter deficiency in the neuropathic urethra. Paraplegia. 1995;33:697-700.

26. Nataluk EA, Assimos DG, Kroovand RL. Collagen injections for treatment of urinary incontinence secondary to intrinsic sphincter deficiency. J Endourol. 1995;9:403-406.

27. Core Outcome Measures in Effectiveness Trials initiative. Available at: www.comet-initiative.org.
28. Abrams P, Andersson KE, Birder L, et al. Fourth international consultation on incontinence recommendations of the international scientific committee: evaluation and treatment of urinary incontinence, pelvic organ prolapse, and fecal incontinence. Neurourol Urodyn. 2010;29:213-240.

29. Abrams P. Clinical Trials in Lower Urinary Tract Dysfunction https://www.ics.org/Documents/Documents.aspx?

DocumentID=41: The International Continence Society 2003.

30. Groen J, Pannek J, Diaz Castro, et al. Summary of european association of urology (EAU) guidelines on neuro-Urology. Eur Urol. 2016;69:324-333.

31. Phe V, Zimmern P, Chartier-Kastler E. Outcome measures for stress urinary incontinence treatment: can we minimally agree? World J Urol. 2015;33:1221-1234.

32. Castillo PA, Espaillat-Rijo LM, Davila GW. Outcome measures and definition of cure in female stress urinary incontinence surgery: a survey of recent publications. Int Urogynecol J. 2010;21: 343-348.

33. Costa P, Perrouin-Verbe B, Colvez A, et al. Quality of life in spinal cord injury patients with urinary difficulties. Development and validation of qualiveen. Eur Urol. 2001;39:107-113.

34. Bonniaud V, Bryant D, Parratte B, Guyatt G. Development and validation of the short form of a urinary quality of life questionnaire: sF-Qualiveen. J Urol. 2008;180:2592-2598.

35. Stoffel JT, Smith G, Crivellaro S, Smith JJ, 3rd, Bresette JF. Selfreported pad use per day reflects patient quality of life after pubovaginal sling surgery. Int Urogynecol J Pelvic Floor Dysfunct. 2009;20:1321-1325.

36. Dylewski DA, Jamison MG, Borawski KM, Sherman ND, Amundsen CL, Webster GD. A statistical comparison of pad numbers versus pad weights in the quantification of urinary incontinence. Neurourol Urodyn. 2007;26:3-7.

37. Farag F, Koens M, Sievert KD, De Ridder D, Feitz W, Heesakkers J. Surgical treatment of neurogenic stress urinary incontinence: a systematic review of quality assessment and surgical outcomes. Neurourol Urodyn. 2016;35:21-25.

38. Tannenbaum C, Corcos J. Outcomes in urinary incontinence: reconciling clinical relevance with scientific rigour. Eur Urol. 2008;53:1151-1161.

\section{SUPPORTING INFORMATION}

Additional Supporting Information may be found online in the supporting information tab for this article.

How to cite this article: Reuvers SH, Groen J, Scheepe JR, et al. Heterogeneity in reporting on urinary outcome and cure after surgical interventions for stress urinary incontinence in adult neurourological patients: A systematic review. Neurourology and Urodynamics. 2018;37:554-565. https://doi.org/10.1002/nau.23364 